\title{
Polyakov loop and QCD thermodynamics from the Landau gauge gluon and ghost propagators
}

\author{
Kouji Kashiwa* \\ $\dagger$ \\ RIKEN/BNL Research Center, Brookhaven National Laboratory, Upton, New York 11973, USA \\ E-mail: kashiwa@ribf.riken.jp
}

\begin{abstract}
In this talk, we present the recent development on the investigation of the deconfinement transition and QCD thermodynamics via the Landau gauge gluon and ghost propagators. The gluon and ghost propagators obtained by the lattice QCD simulation are fitted by the Gribov-Stingl form and then the thermodynamic potential of the gluonic sector is calculated. The Polyakov loop expectation value calculated by minimizing the thermodynamic potential shows a first and second order phase transitions for the pure SU(2) and SU(3) Yang-Mills theories, respectively. Then, thermodynamic quantities are quantitatively agreed with the lattice QCD data near the critical temperature with and without the dynamical quarks. Effects of the quark back reaction to the gluonic sector is also presented.
\end{abstract}

Xth Quark Confinement and the Hadron Spectrum,

October 8-12, 2012

TUM Campus Garching, Munich, Germany

\footnotetext{
* Speaker.

${ }^{\dagger}$ K.K thanks K. Fukushima and Y. Maezawa with whom most of arguments presented here have been developed. The author thanks WHOT-QCD Collaboration for providing us with the gauge configurations.
} 


\section{Introduction}

Understanding of the confinement and deconfinement natures of Quantum Chromodynamics (QCD) is one of the long standing problems in the particle and nuclear physics. To investigate such phenomena, non-perturbative aspects of QCD play an important role.

It was Polyakov [1] who first addressed the deconfinement phase transition successfully in the strong-coupling limit of a pure Yang-Mills theory. The order parameter for deconfinement phase transition was then identified, which is called the Polyakov loop. The strong-coupling expansion was extended to implement quarks and the chiral dynamics [2,3]. One of the most popular approaches to investigate the QCD phase diagram is the chiral effective model (such as the NambuJona-Lasinio model $[4,5,6,7,8,9,10]$, the linear-sigma model [11, 12, 13, 14], etc. [15]) with the Polyakov loop (P-chiral model).

The largest ambiguity in the P-chiral model is the choice of the effective potential of Polyakov loop potential. The initial choice was motivated by the strong coupling expansion [4]. It is used in the P-chiral model and parameters in the potential are fitted with the pure Yang-Mills thermodynamics from the lattice simulation $[4,5,6]$. Since the fitting procedures do not refer to microscopic dynamics at all, it is unclear how the Polyakov loop potential is related or unrelated to non-perturbative characteristics near $T_{c}$.

An important breakthrough came from an attempt to understand quark deconfinement in terms of the Landau-gauge propagators that describe gluon confinement [16, 17]. In the Landau gauge, the deep-infrared enhancement in the ghost propagator causes confinement, while the gluon propagator is infrared suppressed. This behavior is qualitatively consistent with the confinement scenarios by Kugo and Ojima [18] and also by Gribov and Zwanziger [19]. So far, the gluon and ghost propagators in the Landau gauge fixing at zero and finite temperature have been studied in the lattice simulations, the Dyson-Schwinger equation approach, and the functional renormalization group approach [20, 21, 22].

In the talk, we report on an update of Ref. [17] using the state-of-the-art results from the finitetemperature lattice simulation for the gluon and ghost propagators [22]; only the zero-temperature propagators were used in Ref. [17]. We would stress that what we address in this talk is not only an update but should aim to establish a bridge over the common model studies and the first-principle functional approaches. Such a work must be extremely useful for both sides; there are many arguments to suggest that the quark back reaction to the gluonic sector has crucial impacts on the QCD phase-diagram research.

First, we present results obtained in Ref. [23]. In the paper, we discussed the deconfinement transition and QCD thermodynamics with and without the dynamical quarks. Next, the quark back reaction to the gluonic sector is presented. That part is based on Ref. [24].

\section{Formalism}

In the Landau gauge fixing, the inverse gluon propagator can be expressed as

$$
D_{A}^{-1}\left(p^{2}\right)=\left[\left(D_{A}^{T}\right)^{-1} T^{\mu v}+\left(D_{A}^{L}\right)^{-1} L^{\mu v}\right],
$$


where $T^{\mu v}$ and $L^{\mu v}$ are the four-dimensional transverse and longitudinal projection tensors, respectively. At finite $T$, Lorentz symmetry is explicitly broken and then $D_{A}^{T}$ can be further decomposed into the three-dimensional longitudinal and transverse components. Then, three distinct gluon propagators and ghost propagator are appeared; $D_{L}\left(p^{2}\right), D_{T}^{(T)}\left(p^{2}\right), D_{T}^{(L)}$ and $D_{C}$.

To calculate the thermodynamic potential for the gluonic sector, we need the analytic form of the propagators. In this study, we use the Gribov-Stingl from:

$$
D_{L}=\frac{1}{p^{2}}, \quad D_{T}^{(T)}=\frac{c_{t} d_{t}\left(p^{2}+d_{t}^{-1}\right)}{\left(p^{2}+r_{t}^{2}\right)^{2}}, \quad D_{T}^{(L)}=\frac{c_{l} d_{l}\left(p^{2}+d_{l}^{-1}\right)}{\left(p^{2}+r_{l}^{2}\right)^{2}}, \quad D_{C}=\frac{p^{2}+d_{g}^{-1}}{\left(p^{2}\right)^{2}}
$$

Parameters for the pure SU(3) Yan-Mills case at $T=0.86 T_{c}$ are presented in Ref. [22];

$$
\begin{array}{lll}
c_{t}=5.5 \mathrm{GeV}^{2}, & d_{t}=0.152 \mathrm{GeV}^{-2}, & r_{t}^{2}=0.847 \mathrm{GeV}^{2}, \\
c_{l}=3.7 \mathrm{GeV}^{2}, & d_{l}=0.221 \mathrm{GeV}^{-2}, & r_{l}^{2}=0.257 \mathrm{GeV}^{2} .
\end{array}
$$

For the ghost propagator, we use $d_{g}^{-1}=0.454 \mathrm{GeV}^{2}$.

In the leading-order of two-particle irreducible formalism, the thermodynamic potential for the gluonic sector can be expressed as

$$
\mathscr{U}=-\frac{1}{2} \operatorname{tr} \ln D_{A}^{-1}+\operatorname{tr} \ln D_{C}^{-1},
$$

where tr acts on all of indices. The thermodynamic potential finally becomes

$$
\mathscr{U}=\left(\frac{1}{2}-2\right) \mathscr{V}_{\mathrm{pert}}+\frac{1}{2}\left(\mathscr{V}_{A}^{(l)}\left(d_{l}^{-1}\right)-2 \mathscr{V}_{A}^{(l)}\left(r_{l}^{2}\right)\right)+\frac{2}{2}\left(\mathscr{V}_{A}^{(t)}\left(d_{t}^{-1}\right)-2 \mathscr{V}_{A}^{(t)}\left(r_{t}^{2}\right)\right)-\mathscr{V}_{C}\left(d_{C}^{-2}\right)
$$

where $\mathscr{V}_{\text {pert }}, \mathscr{V}_{A}^{(L)}, \mathscr{V}_{A}^{(T)}$ and $\mathscr{V}_{C}$ are the perturbative one-loop [25, 26], three-dimensional longitudinal gluon, three-dimensional transverse gluon and ghost parts, respectively. The explicit form of $\mathscr{V}_{A}$ and $\mathscr{V}_{C}$ is

$$
\mathscr{V}\left(m^{2}\right)=-T \operatorname{tr}_{c} \int \frac{d p}{2 \pi^{2}} \ln \left(1-L_{8} e^{-\beta \sqrt{p^{2}+m^{2}}}\right),
$$

where $\operatorname{tr}_{c}$ is the adjoint color trace and $L_{8}$ is the Polyakov loop operator in the adjoint representation $\left(L_{8}\right)_{a b}=2 \operatorname{tr}_{c}\left(t_{a} L_{3} t_{b} L_{3}\right)$ with fundamental Polyakov loop operator $L_{3}$. At zero chemical potential, the fundamental Polyakov loop, $\Phi=\operatorname{tr}_{\mathrm{c}} e^{i \phi} / 3$, can be simplified as $\Phi=[1+2 \cos (2 \pi a)] / 3$ because the temporal components of the gluon field $\phi$ can be expressed by using one angular parameter $a$; $\phi=2 \pi \times \operatorname{diag}(a,-a, 0)$.

\section{Numerical results}

First, we show the results obtained in Ref. [23]. The parameter fitting result for the gluon propagators is shown in left-panel of Fig. 3. The ghost dressing function is shown in right-panel of Fig. 3. By minimizing the thermodynamic potential (2.5), we can calculate the Polyakov loop expectation value. The result is shown in left-panel of Fig. 3. We find that the critical temperature $T_{c}$ in our approach is $T_{c}=287 \mathrm{MeV}$ which is close to the lattice QCD prediction in the SU(3) Yang-Mills theory $[29,30]$. Thermodynamic quantities, the pressure, the internal energy density 

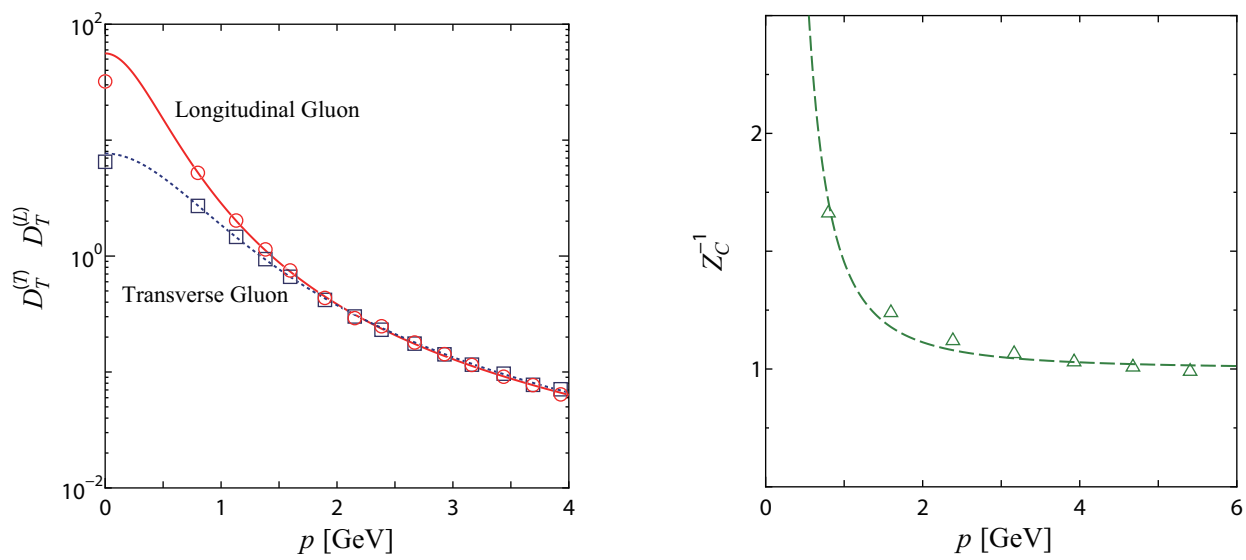

Figure 1: (Left) Three-dimensional transverse and longitudinal gluon propagators $\left(D_{T}^{(L)}\right.$ and $\left.D_{T}^{(T)}\right)$ as a function of the three-dimensional momentum. The symbols represent the lattice data at $T=0.86 T_{c}$ from Ref. [22]. The curves represent the fitting results. (Right) Ghost dressing function at $T=0.84 T_{c}$ from Ref. [22]. The curve is fitting result.
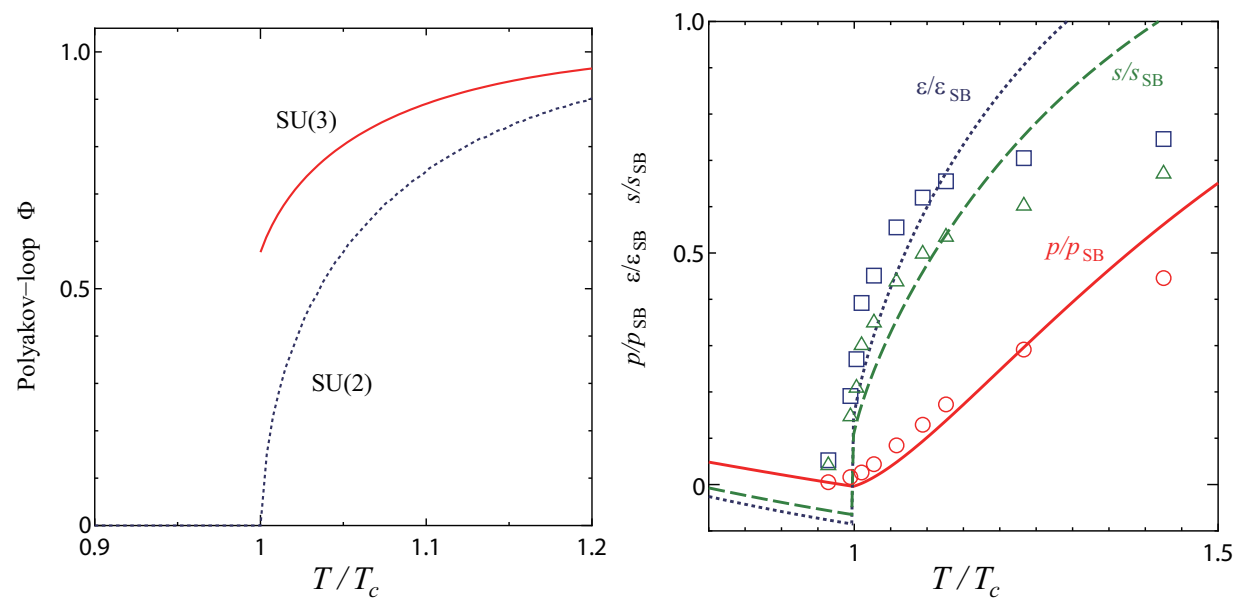

Figure 2: (Left) Polyakov-loop expectation value calculated from the thermodynamic potential for the SU(3) and SU(2) cases with same parameters. (Right) Thermodynamic quantities normalized by the StefanBoltzmann limit for the SU(3) pure Yang-Mills theory. The solid, dotted and dashed lines (the circle, square and triangle symbols) are our results (the lattice QCD data [27]) for the pressure, the internal energy density and the entropy density, respectively.

and the entropy density, are shown in right-panel of Fig. 3. Our result looks consistent with the lattice data neat $T_{c}$. The discrepancy above $T_{c}$ can be understood that we neglect the temperature dependence of the parameters in the propagators. It should be noted that thermodynamic quantities naturally approach the Stefan-Boltzmann limit at high temperature limit.

Next, we show the dynamical quark effects. For the thermodynamic potential of the gluonic sector, we use the pure Yan-Mills one. The quark back reaction to the gluonic sector is discussed in later. The quark contribution of the thermodynamic potential with two-flavor quarks is introduced 

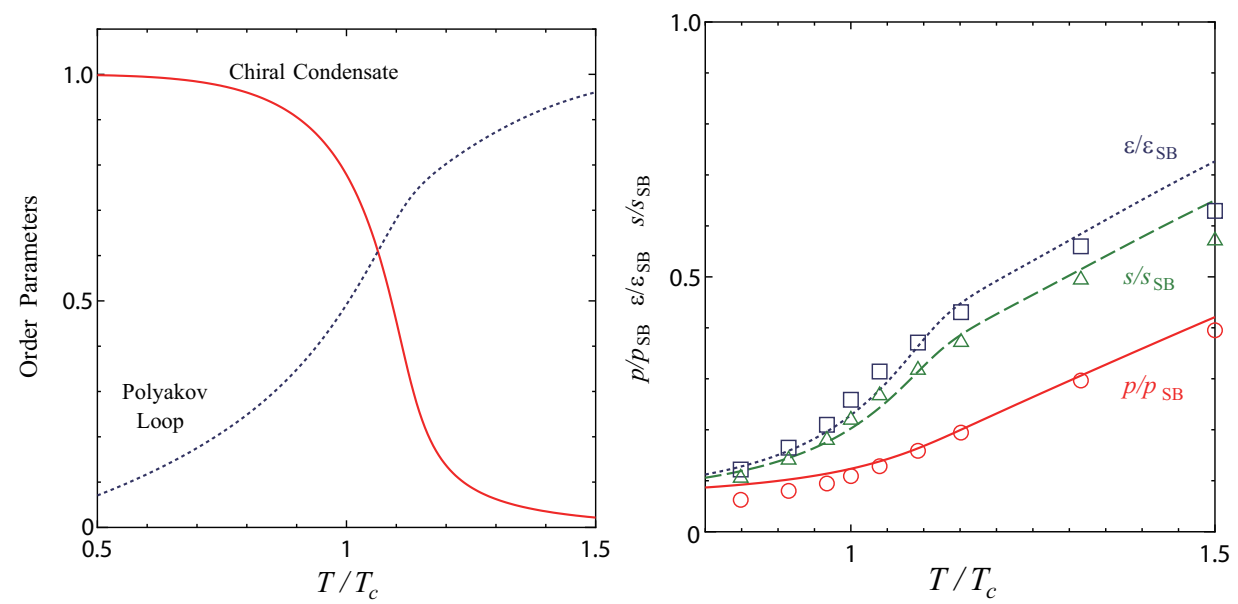

Figure 3: (Left) Polyakov loop expectation value and normalized chiral condensate as a functions of $T$. (Right) Thermodynamic quantities normalized by the Stefan-Boltzmann limit with dynamical quarks. The symbols represent the lattice-QCD results taken from Ref. [28].

by the Nambu-Jona-Lasinio (NJL) model as

$$
\Omega_{\text {quark }}=-4 \int_{\Lambda} \frac{d^{3} p}{(2 \pi)^{3}}\left[N_{c} E_{\mathbf{p}}+T \operatorname{tr}_{\mathrm{c}} \ln \left(1+L_{3} e^{-\beta E_{\mathbf{p}}}\right)\right]+\frac{\left(M-m_{0}\right)^{2}}{2 G},
$$

where $E_{\mathbf{p}}=\sqrt{\mathbf{p}^{2}+M^{2}}$. The parameter set of the NJL part is $\Lambda=631.5 \mathrm{MeV}, G \Lambda^{2}=2.2$ and $m_{0}=$ 5.5 MeV. In the left-panel of Fig. 3, we plot the Polyakov loop expectation value and normalized chiral condensate by minimizing the total thermodynamic potential $\mathscr{U}+\Omega_{\text {quark }}$ where we chose $T_{c}=178 \mathrm{MeV}$ (by an arbitrary criterion that $\Phi$ takes one half there). Thermodynamic quantities, the pressure, the internal energy density and the entropy density, are shown in the right-panel of Fig. 3. Our numerical results are quantitatively agreed with the lattice QCD data near $T_{c}$. We remark that our calculation is two flavors case and the lattice QCD simulation is done in the $(2+1)$ flavors case, but the difference is minor if the quantities are normalized by the Stefan-Boltzmann limit.

Our approach has advantage if we consider the quark back reaction to the gluonic sector because the gluon propagators are explicitly used in the calculation of the thermodynamic potential. The quark back reaction is quite important to understand the QCD phase diagram.

In Ref. [24], we discuss the quark back reaction effect by using the unquenched lattice QCD data. For the lattice QCD simulation, we utilize gauge configurations generated by WHOT-QCD Collaboration on $N_{s}^{3} \times N_{t}=16^{3} \times 4$ lattice with a renormalization-group improved gauge action and a clover improved Wilson quark action with two flavor quarks. The simulations have been performed along the line of constant physics at $m_{\mathrm{PS}} / m_{\mathrm{V}}=0.65$ (ratio of the pseudo-scalar and the vector meson masses at zero temperature). Our results for the gluon propagators are shown in Fig. 4. Figure 4 shows temperature dependence of $D_{T}^{(T)}$ and $D_{T}^{(L)}$ as a function of three-dimensional momentum where propagators are normalized by $T_{\mathrm{pc}}=180 \mathrm{MeV}$. We find that there is significant temperature dependence at infra-red region. On the other hand, the propagators converge at ultraviolet region. When temperature increases, magnitude of both propagators increases at $T<T_{\mathrm{pc}}$. 

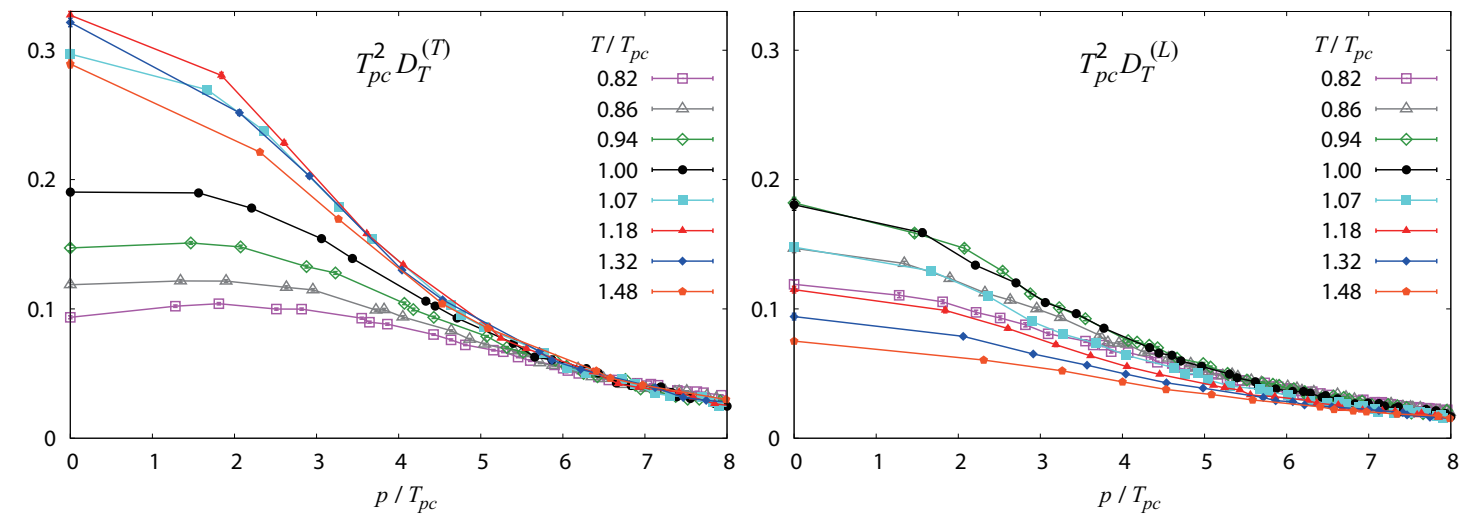

Figure 4: Results of the three-dimensional transverse (left) and longitudinal (right) gluon propagators in three-dimensional momentum space for several temperature. A physical scale is normalized by $T_{\mathrm{pc}}$.

\begin{tabular}{cc}
\hline \hline$T / T_{\mathrm{pc}}$ & $T_{c}^{\prime}[\mathrm{GeV}]$ \\
\hline 0.82 & $0.332(20)(10)$ \\
0.86 & $0.291(26)(11)$ \\
0.94 & $0.233(19)(24)$ \\
\hline
\end{tabular}

Table 1: Results of $T_{c}^{\prime}$ evaluated from thermodynamic potential of the gluonic sector at several $T / T_{\mathrm{pc}}$. The first (second) parenthesis indicates statistical (systematic) errors.

Moreover, we can see the enhancement at $T \sim T_{\mathrm{pc}}$ for $D_{T}^{(L)}$ and $T \sim 1.2 T_{\mathrm{pc}}$ for $D_{T}^{(T)}$. In the case of pure Yan-Mills theory, the gluon propagators are almost insensitive to temperature below $T_{\mathrm{c}}$, whereas these monotonically decrease above $T_{\mathrm{c}}$ [22]. Therefore, the strong sensitivity to $T$ below $T_{\mathrm{pc}}$ in our calculation implies that there are a large number of configurations which can couple to the gluon fields even at $T<T_{\mathrm{pc}}$ where the gluon confinement is slightly broken by the quark back reaction effect. In the calculation of the thermodynamic potential, we also need the ghost propagator. Here, we utilize results of $D_{C}$ obtained in Ref. [23] by fitting the lattice QCD data in pure Yan-Mills theory [22] because the ghost propagator is not sensitive to the dynamical quarks due to lack of the quark-ghost vertex [31].

The gluon propagators shown in Fig. 4 are also fitted by the Gribov-Stingl form in the case of pure Yan-Mills theory. Then the critical temperature of the thermodynamic potential $T_{c}^{\prime}$ can be estimated. It should be noticed that $T_{c}^{\prime}$ does not correspond to $T_{\mathrm{pc}}$ because it is obtained by the purely gluonic sector. Qualitative shape of the thermodynamic potential (2.5) is the same with the case of pure Yan-Mills theory [23]. Therefore, it exhibits the first order phase transition. Contribution of the quark back reaction appears as a shift of the characteristic scale which relates $T_{c}^{\prime}$ in the effective approach. Table 3 summarizes results of $T_{c}^{\prime}$ in $\mathrm{GeV}$ unit for several $T / T_{\mathrm{pc}}$. Parameters in the Gribov-Stingl form and the explanation of the error estimation is shown in Ref. [24]. We can see that $T_{c}^{\prime}$ is sensitive to input $T / T_{\mathrm{pc}}$ and decreases with $T$ increasing at $T<T_{\mathrm{pc}}$. Since the number of lattice configurations which couple to the gluon fields increases with increasing $T$ below $T_{\mathrm{pc}}$, the 
decrease of $T_{c}^{\prime}$ is caused by the quark back reaction via the gluon propagators.

\section{Summary}

In this talk, we present the recent development on the investigation of the deconfinement transition and QCD thermodynamics via the gluon and ghost propagators. This work is the extension of the idea of Ref. [17].

The gluon and ghost propagators at finite $T$ in the Landau gauge measured in the lattice simulation were fitted by the Gribov-Stingl form. We took the fitting parameters of propagators at finite $T$ from Ref. [22] and found an easy way to calculate the pressure, the internal energy density and the entropy density as well as the order parameters as functions of $T$ in the pure Yan-Mills theory and the system with dynamical quarks. The dynamical quark effects were investigated by using the NJL model. Our numerical results are quantitatively agreed with the lattice QCD data near $T_{c}$.

The quark back reaction effect was investigated in the same procedure in the case without the quark back reaction by considering the unquenched lattice QCD data. We have calculated the gluon propagators of the three-dimensional longitudinal and traverse sectors in lattice QCD simulations with two-flavor dynamical quarks at finite temperature. The simulations have been performed by utilizing gauge configurations generated by WHOT-QCD Collaboration on $N_{s}^{3} \times N_{t}=16^{3} \times 4$ lattice with a renormalization-group improved gauge action and a clover improved Wilson quark action with two flavor quarks The simulations have been performed along the line of constant physics at $m_{\mathrm{PS}} / m_{\mathrm{V}}=0.65$. We obtained that the magnitude of the gluon propagators increases at $T<T_{p c}$. This can be regarded as contributions of the quark back reaction since the gluon propagators are almost insensitive below the critical temperature in the pure Yan-Mills theory [22]. In order to estimate effects of the quark back reaction to the gluonic sector, we fit the propagators by GribovStingl form and input these to the thermodynamic potential calculation. The critical temperature estimated from the purely gluonic sector decreased with temperature of the gluon propagators increasing at $T<T_{p c}$. This means that the enhancement of the quark back reaction reduces the critical temperature of the gluonic sector.

\section{References}

[1] A. M. Polyakov, Phys. Lett. B 72, 477 (1978).

[2] E. -M. Ilgenfritz and J. Kripfganz, Z. Phys. C 29, 79 (1985).

[3] A. Gocksch and M. Ogilvie, Phys. Rev. D 31, 877 (1985).

[4] K. Fukushima, Phys. Lett. B 591, 277 (2004) [hep-ph/0310121].

[5] C. Ratti, M. A. Thaler and W. Weise, Phys. Rev. D 73, 014019 (2006) [hep-ph/0506234].

[6] S. Roessner, C. Ratti and W. Weise, Phys. Rev. D 75, 034007 (2007) [hep-ph/0609281].

[7] W. -j. Fu, Z. Zhang and Y. -x. Liu, Phys. Rev. D 77, 014006 (2008) [arXiv:0711.0154 [hep-ph]].

[8] M. Ciminale, R. Gatto, N. D. Ippolito, G. Nardulli and M. Ruggieri, Phys. Rev. D 77, 054023 (2008) [arXiv:0711.3397 [hep-ph]].

[9] Y. Sakai, K. Kashiwa, H. Kouno and M. Yahiro, Phys. Rev. D 77, 051901 (2008) [arXiv:0801.0034 [hep-ph]]. 
[10] K. Fukushima, Phys. Rev. D 77, 114028 (2008) [Erratum-ibid. D 78, 039902 (2008)] [arXiv:0803.3318 [hep-ph]].

[11] B. -J. Schaefer, J. M. Pawlowski and J. Wambach, Phys. Rev. D 76, 074023 (2007) [arXiv:0704.3234 [hep-ph]].

[12] B. -J. Schaefer, M. Wagner and J. Wambach, Phys. Rev. D 81, 074013 (2010) [arXiv:0910.5628 [hep-ph]].

[13] T. K. Herbst, J. M. Pawlowski and B. -J. Schaefer, Phys. Lett. B 696, 58 (2011) [arXiv:1008.0081 [hep-ph]].

[14] B. J. Schaefer and M. Wagner, Phys. Rev. D 85, 034027 (2012) [arXiv:1111.6871 [hep-ph]].

[15] E. Megias, E. Ruiz Arriola and L. L. Salcedo, Phys. Rev. D 74, 114014 (2006) [hep-ph/0607338].

[16] J. M. Pawlowski, D. F. Litim, S. Nedelko and L. von Smekal, Phys. Rev. Lett. 93, 152002 (2004) [hep-th/0312324].

[17] J. Braun, H. Gies and J. M. Pawlowski, Phys. Lett. B 684, 262 (2010) [arXiv:0708.2413 [hep-th]].

[18] T. Kugo, hep-th/9511033.

[19] D. Zwanziger, Nucl. Phys. B 412, 657 (1994).

[20] N. Vandersickel and D. Zwanziger, Phys. Rept. 520, 175 (2012) [arXiv:1202.1491 [hep-th]].

[21] C. S. Fischer, A. Maas and J. A. Muller, Eur. Phys. J. C 68, 165 (2010) [arXiv:1003.1960 [hep-ph]].

[22] R. Aouane, V. G. Bornyakov, E. M. Ilgenfritz, V. K. Mitrjushkin, M. Muller-Preussker and A. Sternbeck, Phys. Rev. D 85, 034501 (2012) [arXiv:1108.1735 [hep-lat]].

[23] K. Fukushima and K. Kashiwa, arXiv:1206.0685 [hep-ph].

[24] K. Kashiwa and Y. Maezawa, arXiv:1212.2184 [hep-ph].

[25] D. J. Gross, R. D. Pisarski and L. G. Yaffe, Rev. Mod. Phys. 53, 43 (1981).

[26] N. Weiss, Phys. Rev. D 24, 475 (1981).

[27] S. Datta and S. Gupta, Phys. Rev. D 82, 114505 (2010) [arXiv:1006.0938 [hep-lat]].

[28] S. Borsanyi, G. Endrodi, Z. Fodor, A. Jakovac, S. D. Katz, S. Krieg, C. Ratti and K. K. Szabo, JHEP 1011, 077 (2010) [arXiv:1007.2580 [hep-lat]].

[29] G. Boyd, J. Engels, F. Karsch, E. Laermann, C. Legeland, M. Lutgemeier and B. Petersson, Nucl. Phys. B 469, 419 (1996) [hep-lat/9602007].

[30] O. Kaczmarek, F. Karsch, P. Petreczky and F. Zantow, Phys. Lett. B 543, 41 (2002) [hep-lat/0207002].

[31] E. -M. Ilgenfritz, M. Muller-Preussker, A. Sternbeck, A. Schiller and I. L. Bogolubsky, Braz. J. Phys. 37, 193 (2007) [hep-lat/0609043]. 